\title{
REMARK ON A THEOREM OF FROBENIUS ${ }^{1}$
}

\author{
R. H. SHAW
}

1. Introduction. A finite group $G$ will be called a Frobenius group with respect to one of its subgroups $H$ if:

a. $H$ is its own normalizer (is ipsonormal) in $G$; and

b. the intersection of $H$ with any of its conjugates in $G$ is the identity element $E$.

It is an unsolved problem of group theory to prove, without using group characters, the following theorem of Frobenius: If $G$ is a Frobenius group with respect to $H$, then the set $N$ consisting of the identity element and those elements of $G$ not in $H$ or its conjugates is a normal subgroup of $G$. The purpose of this note is to establish the theorem for solvable $H$, without using characters but instead by applying a transfer.

2. Application of transfers. If $g$ is an element of a group $G$, then the mapping

$$
g \rightarrow V_{G \rightarrow H}(g)=H^{\prime} \prod_{i=1}^{r} g_{i} g\left[\left(g_{i} g\right)^{*}\right]^{-1}
$$

(where $G=H \cup H g_{2} \cup \ldots \cup H g_{r}, H^{\prime}$ is the first derived group of $H$, and $x^{*}$ denotes the representative of the right coset containing $x$ in the given decomposition) carries $G$ into $H / H^{\prime}$ and is called the transfer of $G$ into $H$. It is shown in Chapter $\mathrm{V}$ of Zassenhaus ${ }^{2}$ that transfer is independent of the choice of representatives and that transfer is homomorphic. These properties are easily established by direct calculation not involving matrices because of the fact that commutator quotient groups are abelian.

In view of this it is now possible to establish the following theorem.

THEOREM. If $G$ is a Frobenius group with respect to a solvable subgroup $H$, then $N$ is a normal subgroup of $G$.

PRoof. Let $H$ be $k$-step metabelian. Decompose $G$ into right cosets of $H$ :

Received by the editors February 29, 1952.

${ }^{1}$ This note is the result of a discussion arising in the author's final examination for the degree of Doctor of Philosophy at The George Washington University. Acknowledgment is made to Professor F. E. Johnston of that university and to Professor Marshall Hall, Jr., of The Ohio State University.

2 Hans Zassenhaus, The theory of groups (trans. by Saul Kravetz), New York, Chelsea, 1949. 


$$
G=H+H g_{2}+H g_{3}+\cdots+H g_{r} .
$$

Transfer $G$ to $H$ by the mapping $V_{1}$. Consider any element $h$ of $H$. The factor contributed to $V_{1}(h)$ by the coset $H$ is $E h E^{-1}=h$. So $V_{1}(h)=H^{\prime} h \prod_{i=1}^{s} g_{a_{i}} h^{P i} g_{a_{i}}^{-1}$. But $g_{a_{i}} \in H$, and $g_{a_{i}} h^{P i} g a_{a_{i}}^{-1} \in H$. Therefore $g_{a_{i}} h^{P{ }^{i}} g_{a_{i}}^{-1}=E$, and $V_{1}(h)=H^{\prime} h$ for all $h \in H$. In particular, $V_{1}(E)$ $=H^{\prime}$, and $V_{1}\left(h^{\prime}\right)=H^{\prime}$ for all $h^{\prime} \in H^{\prime}$.

Now consider an element of a conjugate of $H$, say $m^{-1} h m, h \in H$, $m \in G-H$. The factor contributed to $V_{1}\left(m^{-1} \mathrm{hm}\right)$ by the coset $H m$ is $m m^{-1} h m m^{-1}=h$. So

$$
V_{1}\left(m^{-1} h m\right)=H^{\prime} h \prod_{i=1}^{t} g_{a_{i}}\left(m^{-1} h^{q} m\right) g a_{i}^{-1} .
$$

But $g a_{i} \notin H m$. Therefore every $g_{a_{i}}\left(m^{-1} h^{\prime i} m\right) g_{a_{i}}^{-1}=E$, and $V_{1}\left(m^{-1} h m\right)$ $=H^{\prime} h$ for all $h \in H, m \in G-H$. For those elements $h^{\prime} \in H^{\prime}$,

$$
V_{1}\left(m^{-1} h^{\prime} m\right)=H^{\prime} \text {. }
$$

Now consider an element $n$ of the set $N$ consisting of $E$ and those elements of $G$ not in $H$ or its conjugates. Take $n \neq E$.

$$
V_{1}(n)=H^{\prime} \prod_{i=1}^{w} g_{a_{i}} n^{r i} g_{a_{i}}^{-1}
$$

Each factor $g_{a_{i}} n^{r} g_{a_{i}}^{-1}$ lies in $H$, by construction.

But every power of an element of $N$, except for a multiple of the order of that element, lies in $N$, but not in $H$ or its conjugates; and all conjugates of elements of $N$ lie in $N$.

Hence each factor is the identity, and

$$
V_{1}(n)=H^{\prime} \quad \text { for all } n \in N .
$$

Now $V_{1}$ defines a homomorphism of $G$ onto $H / H^{\prime}$. Its kernel is the group $Q_{1} \subset G$ :

$$
Q_{1}=N+\sum_{m \in \sigma} m^{-1} H^{\prime} m, \quad \text { each element counted once. }
$$

If $k=1$, then $H^{\prime}=E$, and the theorem is proved.

If $k>1, H^{\prime} \supset H^{\prime \prime}$, and so $Q_{1} \supset H^{\prime \prime}$.

Transfer $Q_{1}$ to $H^{\prime}$ by the mapping $V_{2}$. By exactly similar argument, this gives rise to the group

$$
Q_{2}=N+\sum_{m \in Q_{1}} m^{-1} H^{\prime \prime} m, \quad \text { each element counted once, }
$$

and $Q_{2}$ is a subgroup of $Q_{1}$ and a fortiori of $G$. 
But $H$ is solvable and finite, and so $k$ is finite. At length, by iteration, we arrive at

$$
Q_{k}=N \text {, }
$$

which is therefore a subgroup of $G$. Since $N$ is a normal set in $G$, the theorem is proved.

ArLington, Virginia 